\title{
Guiding students towards an understanding of the electromotive force concept in electromagnetic phenomena through a teaching-learning sequence
}

\author{
Kristina Zuza $\odot,{ }^{1}$ Mieke De Cock $\odot,{ }^{2}$ Paul van Kampen $\odot,{ }^{3}$ Thomas Kelly, ${ }^{4}$ and Jenaro Guisasola ${ }^{1}$ \\ ${ }^{1}$ Department of Applied Physics \& DoPER, University of the Basque Country (UPV/EHU), \\ San Sebastian E-20018, Spain \\ ${ }^{2}$ Department of Physics and Astronomy \& LESEC, KU Leuven, Leuven B-3000, Belgium \\ ${ }^{3}$ Centre for the Advancement of STEM Teaching and Learning \& School of Physical Sciences, \\ Dublin City University (dcU), Dublin 9, Ireland \\ ${ }^{4}$ Department of Computer Science \& Applied Physics, School of Science \& Computing, \\ Galway-Mayo Institute of Technology, Galway, Ireland
}

(Received 12 April 2020; accepted 14 July 2020; published 3 August 2020)

\begin{abstract}
In this work we present the application of design based research (DBR) methodology to conduct a systematic iterative study of the design and implementation of a teaching-learning sequence (TLS) on emf (electromotive force). This work is the final part of a broader study that started with the analysis of students' difficulties with emf in the contexts of transitory current, direct current, and electromagnetic induction. We complete our research by investigating to what extent students see emf as a cross-cutting concept. We establish the design parameters based on an epistemological analysis and the empirical data, and describe how we devised and implemented the first version of the TLS that takes $4.5 \mathrm{~h}$ of classroom time. We analyze the data obtained in the first implementation and describe the redesign process that resulted in a second version of the TLS that takes $1.5 \mathrm{~h}$ of classroom time. We conclude that both versions of the TLS effect a similar boost in student attainment of learning objectives compared to the control group.
\end{abstract}

DOI: 10.1103/PhysRevPhysEducRes.16.020110

\section{INTRODUCTION}

Some topics are included in introductory physics curricula all around the world. These topics include direct current (dc) circuits and electromagnetic induction (EMI) [1-3]. They are part of the basic knowledge of science and engineering graduates and fundamental to understanding today's technology. There is a body of research that shows students' difficulties with learning these topics [4-7] and research-based proposals to overcome them [8-11].

Comparatively little research has been published on students' understanding of electromotive force (emf), a concept that is fundamental to understanding both dc circuits and EMI phenomena [12,13]. Moreover, the emf concept is a good example to illustrate the internal consistency of scientific theories, and how a scientific concept has the same meaning independent of the context in which it is used. Previous studies concluded that emf is a complex concept for introductory physics students and that they have serious difficulties in understanding it, even if they are able to compute the value of emf and give correct answers

Published by the American Physical Society under the terms of the Creative Commons Attribution 4.0 International license. Further distribution of this work must maintain attribution to the author(s) and the published article's title, journal citation, and DOI. to plug and chug exercises $[6,14,15]$. We will describe the details of this research below.

In this paper, we present teaching-learning sequences (TLS) that guide students to make sense of the emf concept. The development of TLSs is an important line of research in science education that began in the early 1990s. It focuses on the design and evaluation of curricular products that include sequences of activities that aim to improve student learning of specific topics at a small to medium scale (for example, a small number of teaching sessions or a complete sequence of lessons on a particular topic), but not at the large-scale level of several modules or even a complete program. The literature refers to this kind of teaching activities as TLSs [16-18]. A distinctive feature of a TLS is that the material includes well-researched teaching-learning activities that have been empirically adapted to students' needs [19].

The study presented here is the second part of a research project that started from an analysis of the epistemological meaning of the emf concept and investigated to what extent students understood this after lectures and tutorials on the subject. We used a number of key characteristics of emf obtained from this analysis as a standard to gauge students' attainment in introductory university physics courses. In the first part of this study, we identified students' difficulties with the emf concept in transitory currents, simple resistive dc circuits, and EMI [14,15]. 
We found that very few students show that they think of emf as the work done per unit charge by a nonconservative force. Some students use science-based arguments without really showing they know the difference between emf and potential difference. These students say that emf moves the electrons in de circuits [14] or that a changing magnetic flux generates an emf in EMI circuits, but there is nothing about the relation between emf and potential difference in their answers [15]. In both cases students argue that current generates potential difference, based on Ohm's law.

In de circuits we find students arguing that potential difference between the terminals of the battery is conceptually the same as the battery emf. Other students ascribe some properties of emf to potential difference; e.g., they may say that the potential difference between the battery terminals is independent of the current through the battery. The research has also found students claiming that potential difference generates emf or equating Ohm's law with the energy balance equation [14]. Likewise, in the context of EMI students reason that potential difference is due to a change in flux, that if there is no current there is no potential difference, or that currents are generated by static magnetic fields [15].

The aim of this second part of the study is the development and implementation of a TLS, based on these results, that includes activities that guide students to develop an understanding of the meaning of emf in different contexts and help them overcome the difficulties described above.

The development of the TLSs was founded in the methodology of design based research (DBR), which allows us to make explicit the relations between the theoretical elements we assume and the decisions we made in the design and evaluation of the TLSs $[20,21]$. The DBR methodology for developing teaching materials proposes an iterative process comprising three principal steps: design, implementation, and retrospective analysis or evaluation [19]. We will explain these three steps in the context of our TLSs.

In Sec. II, we establish the basis of the TLS design informed by the definition of the learning objectives and the students' difficulties identified in previous studies. In Sec. III we present the activities and the implementation of two versions of a TLS. In Sec. IV the results of the implementations, the evaluation, and effectiveness of the TLSs are presented. Discussion and conclusions are presented in Sec. V.

\section{TLS DESIGN: ESTABLISHING THE PARAMETERS}

In this section, we first describe the educational context in which the TLS was implemented. We then summarize the three main factors in the design of the TLS: defining the key characteristics of emf, analyzing students' difficulties with understanding these, and defining learning objectives.

\section{A. Educational context}

The TLS was implemented in a transformed calculusbased introductory physics course for first-year engineering students at the University of the Basque Country (UPV/ EHU). All students have a similar background in physics (3 semesters of high school physics on the topics of classical mechanics, electromagnetism and modern physics), and they have passed an external examination to qualify for studying science at university. Courses at UPV/EHU run for 15 weeks.

It is important to note that the intervention we describe in this paper is about changes made to an already existing transformed course format. In the transformed physics course, the teaching strategy involves posing problems or "situations" to students, with directions that guide them to find solutions. The solution must be achieved in accordance with scientific practices we want the student to become familiar with $[10,22,23]$. The transformed calculus-based physics course provides an interactive learning context in which

(i) Students are organized in cooperative groups of three or four.

(ii) The TLS is structured around several problems whose resolution helps students attain the desired learning objectives.

(iii) In each problem, there is a number of activities that guide students to solve the problem. They have opportunities to make argued decisions in the small group.

(iv) All ways of solving the task are discussed, guided by the teacher, and a classroom summary is formulated.

\section{B. Emf: A cross-cutting concept}

Nowadays, there is consensus that understanding concepts and theories requires knowing the context of the inquiry in which they were built and developed as well as the resulting body of knowledge [24,25]. The development of scientific concepts and theories does not only require creativity but also a systematic process of problem solving and initial hypothesis testing [26]. Consequently, knowing the epistemological elements that underlie the meaning of a concept can provide important information to defining its key characteristics. In introductory physics courses students and instructors often talk informally about "the circuit" when referring to the network of all components except the battery (as in, "the circuit is connected to a battery") [27]. However, it is crucial to consider the battery as part of the circuit to understand the energy balance in a circuit and to distinguish between emf and potential difference in a familiar context of an electric circuit is an important learning objective in the introductory physics program at university [9]. In the battery a series of "nonconservative actions" takes place through which the charges move between the terminals inside the battery. The emf quantifies these actions as the "work done" per unit charge or the electric energy delivered per unit charge to 
produce and maintain the electric potential difference that allows an electrical current to flow. Thus the emf pertains to a work done by a non-Coulombic force to move a charge between the terminals inside the battery; potential difference to a work done by a Coulombic electric field to move a unit of charge between the terminals outside the battery $[13,28]$. In particular, in order to simplify the analysis of the battery for students to understand the nonconservative actions, we use the analogy of a "mechanical battery" which has a conveyor belt driven by a motor (nonCoulombic forces) that pulls electrons out of one plate, making it positive, and pushes them onto another plate, making it negative [29]. In EMI circuits the changing magnetic field or the movement of conductors in magnetic fields are likewise related to nonconservative forces doing work. If the EMI circuit is closed there is no charge accumulation at any point, and hence there is no potential difference between any two points of the circuit [30]. Many textbooks distinguish between potential difference and open source voltage, and in doing so implicitly (but rarely explicitly) acknowledge that a potential difference does not necessarily set up a current. In EMI circuits, it is rarely emphasized that a non-Coulombic electric field and emf are induced even in the absence of current [15].

To understand the role of the emf in any circuit and to distinguish it from related concepts such as potential difference, it is useful to analyze the energy balance in the circuit. Considering the energy balance gives consistency not only to the emf concept but also to the generalized principle of work and energy embodied in Kirchhoff's second law [14,15,31,32].

Based on the above, we define six key characteristics for properly understanding the concept of emf:

K1. Emf is the work done per unit charge by a nonconservative force.

$\mathrm{K} 2$. Emf is not the same as potential difference, even when they have the same numerical value. Potential difference is the work done per unit charge by a conservative force, as it is the difference in potential energy per unit charge between two points.

K3. In dc circuits, emf is confined to the battery. Emf produces a charge separation between the terminals of the battery which results in a potential difference. In closed EMI circuits there is no charge separation and the emf is nonzero wherever the magnetic flux is changing.

K4. Even though the sources of emf are different in dc circuits (battery) and EMI circuits (changing magnetic field or moving conductor in a magnetic field), the emf concept is consistent across these contexts.

$\mathrm{K} 5$. The presence of an emf does not imply the presence of current in a circuit (e.g., open circuit, nonconducting path, ...)

K6. The application of the generalized principle of work and energy (the energy balance of the circuit) per unit charge may be equated with Kirchhoff's second law (the "loop rule").

\section{Summary of students' difficulties with emf}

In the design phase of developing the TLS using DBR methodology, we take into account the students' difficulties with key characteristics of emf we studied earlier $[14,15]$. The detection of the difficulties in dealing with emf was done in two parts. The first part concerned transitory and dc circuits [14]. The second study of this project investigated students' difficulties with emf in EMI contexts [15]. In each study, the research instrument used was an original questionnaire designed by the authors. Open-ended questions were designed, each asking for a careful explanation. The questionnaires were validated in terms of content and teaching aims before they were administered by experienced physics faculty members from each university, who answered the questionnaires and made suggestions that were taken into account in the final writing of the questionnaires. Furthermore, some of the questions asked about concepts in settings commonly used in numerical end-ofchapter exercises, so students are likely to be familiar with the context of the questions. Moreover, in-depth discussions with students after they had taken the questionnaire confirmed that there were generally no problems with interpreting the questions, i.e., the students had understood what they had been asked to do.

The answers from the questionnaires were carefully analyzed by the authors of the studies. In each study, one of the authors performed a preliminary analysis in which the answers were grouped according to the explanations given by students. The criteria to group the answers into categories were (a) each category should be clearly related to research phenomena, so that each one tells us something distinct about a particular way of experiencing the phenomenon; (b) categories must be hierarchical, in other words, they must progress from simple to complex relations [33]. The categories of answers that emerged were then discussed in a meeting in which each author analyzed a sample representing $10 \%$ of the questionnaires. The original categories were redefined until a consensus was reached. Each researcher then analyzed each questionnaire individually. Finally, a meeting was held in which all the answers were classified and a consensus was reached on the responses where there had been some disagreement.

This first study found that students' answers to open ended questions about emf could be grouped into four general categories regardless of the university they attended (Universidad Pedagógica Nacional, Colombia; UPV/EHU, Spain; KU Leuven, Belgium). In the second study, we found the same general categorization could be applied to responses from students from three universities (with students from DCU, Ireland instead of Universidad Pedagógica Nacional). Table I summarizes the general categories illustrated with examples for each context. 
TABLE I. Summary of arguments used by introductory physics students to explain open ended questions dealing with emf [14,15].

\begin{tabular}{|c|c|c|}
\hline Categorization of answers & Transitory or de circuits & EMI circuits \\
\hline A. Correct understanding of emf & $\begin{array}{l}\text { - The emf is the work done per unit charge } \\
\text { by a nonconservative force }\end{array}$ & \\
\hline $\begin{array}{l}\text { B. Isolated elements from a } \\
\text { scientific framework }\end{array}$ & - The emf moves the electrons & $\begin{array}{l}\text { - There is emf due to a changing magnetic } \\
\text { flux } \\
\text { - As long as a magnet is moving there will be } \\
\text { a current in the loop. } \\
\text { - The conductor is moving within a magnetic } \\
\text { field so a potential difference will } \\
\text { appear between ends }\end{array}$ \\
\hline $\begin{array}{l}\text { C. Emf and potential difference } \\
\text { are mixed up }\end{array}$ & $\begin{array}{l}\text { - Potential difference between the terminals } \\
\text { of the battery and emf are the same concepts } \\
\text { - Potential difference between terminals } \\
\text { of a battery remains constant } \\
\text { - Potential difference generates emf } \\
\text { - Ohm's law is the energy balance equation }\end{array}$ & $\begin{array}{l}\text { - There is potential difference due } \\
\text { to a change in flux } \\
\text { - If there is no induced potential } \\
\text { difference, there is no current }\end{array}$ \\
\hline $\begin{array}{l}\text { - Scientific characteristics } \\
\text { are misapplied }\end{array}$ & $\begin{array}{l}\text { - If there is a current, Ohm's law implies } \\
\text { there must be a potential difference }\end{array}$ & $\begin{array}{l}\text { - If there is a current, Ohm's law } \\
\text { implies there must be a potential } \\
\text { difference } \\
\text { - Current is generated by magnetostatic fields }\end{array}$ \\
\hline
\end{tabular}

We inferred from our previous research that students experience several difficulties, and that each key characteristic has a related difficulty. Students have difficulties in understanding the nonconservative nature of the emf and defining it as the work per unit charge (K1). The difficulty in distinguishing emf and potential difference is the most frequent (K2). Furthermore, students have problems identifying the source of emf (K3), think the presence of an emf implies the presence of a current (K5), and reason as if Ohm's law represents the energy balance of the circuit (K6).

Key characteristic K4 was not investigated in our previous research. To close this gap and cover all the defined key characteristics we designed a question that probes whether students understand emf as the same concept independent of context (see Appendix question Q2). Students in three universities [DCU (30 students), UPV/EHU (89 students), and KU Leuven (100 students)] were given the question under the same examlike conditions as in Refs. [14,15], and we followed the same research methodology for validity of the question and reliability of the analysis. The question asks students to explain whether the electromotive force quantity in the context of electrical circuits and in the EMI phenomena correspond to the same physical meaning (see Q2 in the Appendix). The goal of the question is to analyze whether students recognize emf as a consistent cross-cutting concept independent of the context. We found that students also have difficulties related with key characteristic K4. Less than $10 \%$ of students (5\% DCU, 15\% UPV/EHU, and $0 \% \mathrm{KU}$ Leuven) reasoned that meaning of emf is not context dependent. For this reason, the TLS design should take into account difficulties with all characteristics.

\section{A. Defining learning objectives}

Having decided on the content, the next step in the design of the TLS is the definition of the learning

TABLE II. The relation between learning objectives and key characteristics is presented.

\begin{tabular}{lc}
\hline \hline Learning objectives. Students will be able to & Key characteristic \\
\hline LO1. Define emf as work done per unit charge by a nonconservative & $\mathrm{K} 1, \mathrm{~K} 2$ \\
force found in a variety of sources. & $\mathrm{K} 4$ \\
LO2. Recognize that emf is a cross-cutting concept that has the same physical \\
meaning in different contexts such as a battery in dc circuits or EMI circuits. \\
$\begin{array}{l}\text { LO3. Recognize that potential difference only exists when charges have been separated. } \\
\text { LO4. Distinguish between potential difference and emf. }\end{array}$ \\
$\begin{array}{l}\text { LO5. Analyze the energy balance of the circuit, identify whether the force doing } \\
\text { the work is conservative or nonconservative. }\end{array}$ \\
LO6. Demonstrate that the presence of an emf does not imply the presence of a current. \\
\hline
\end{tabular}


objectives. Then, the structure of the TLS and the TLS itself will be presented. Regarding all the aforementioned, we present in Table II the learning objectives we would like the students to attain in the TLS and how they relate to the key characteristics.

\section{TLS DESIGN: DEVISING AND IMPLEMENTING STUDENT ACTIVITIES}

Once the learning objectives were defined, we developed and tested a sequence of activities for implementation in the classroom during regular teaching of a transformed calculus based physics course. We used a quasiexperimental post-test design. The control group comprises the cohorts of UPV/EHU students in our previous work. They experienced a transformed course format that comprised interactive lectures on emf but not any tutorial targeting student understanding of the topics. The groups that followed TLS1 (2014-2015) and TLS2 (2015-2016 and 2016-2017) form the three experimental groups [34]. As we will explain later, we implement two versions of the TLS: TLS1 (first version) and TLS2 (second version after redesign).

All groups that took the transformed course used the same syllabus as the students in the traditional course. The transformed course did not take additional time. In both course formats, the dc circuits and electromagnetic induction syllabus incorporates elements included in the course textbook [35]. In the transformed course format there were two hours of lecture classes with an enrolment of 50-60 students, and a $1.5 \mathrm{~h}$ problem-solving session in which the group is split in half, in each of the 15 weeks. Thus, in what follows, we describe the effects of developing a targeted intervention within an already transformed setting.
This intervention took the form of two TLSs, since after a first implementation and evaluation (TLS1), we redesigned the activities and put together a second version of the TLS (TLS2). For clarity of presentation, we first present both TLSs before giving a detailed discussion of the results obtained after each implementation. While this ordering does not reflect the principles of DBR, it avoids a lot of repetition.

\section{A. First version of the TLS (TLS1)}

As we explained in Sec. II. A, through the interactive teaching strategies that we use in the classroom, we emphasize the integration of understanding of scientific concepts and we encourage students to engage in scientific practices. To make scientific conceptions more attractive and fruitful for students than spontaneous conceptions, there must be repeated and lasting opportunities for students to put into practice procedures and criteria of testing and acceptance that are characteristic of scientific work. In this work they can be introduced to the "scientific culture"- as far as possible in teaching.

In our approach the teacher has an essential role in posing problems and in guiding both their resolution and the learning process. The students are not explicitly confronted with the alternative ideas they may have [36]; instead, they are given the opportunity to modify or refine their ideas in the light of guided questions. For this reason we call the approach "teaching and learning as an oriented research activity" [19]. For the resolution of the driving problems, the class is divided into small working groups (3-4 students) that develop a preliminary inquiry. In each guiding problem, there is a number of activities that

\section{Charged particle in a closed loop}

A particle with charge $q$ moves along a continuous path EFGHE. The particle has total energy $U$ each time it passes point $F$.

A. Is it possible that conservative forces act on the particle? Explain.

Is it possible that non-conservative forces act on the particle?

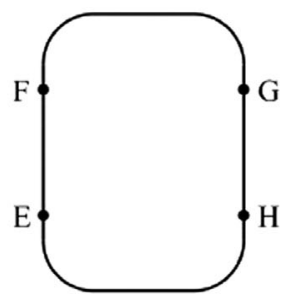
Explain.

A change is now made so that the particle loses an amount of energy $\Delta U_{1}$ on the path GH. A change is also made to the path $\mathrm{EF}$ so that every time the particle passes point $\mathrm{F}$, it has the same energy $U$.

B. Is it possible that all forces acting on the particle on path $\mathrm{GH}$ are conservative? Explain.

Is it possible that all forces acting on the particle on path EF are conservative? Explain.

FIG. 1. Second activity of the first problem. 
guide students to solve the problem. Students discuss and work out activities in their small group. Then, group answers are pooled and analyzed by the students and the teacher, coming to a reasoned consensus. During group work, the teacher's role is to encourage and guide students, to question their answers and to make them think about it or provide additional information if necessary.

TLS1 was constructed based on these principles. It took the form of a sequence of three guiding problems. Each problem took $1.5 \mathrm{~h}$ in class $(4.5 \mathrm{~h}$ in total). The first guiding problem was designed to be implemented after completing existing instruction on electrostatics, the second after dc circuits, and the third after EMI. The three guiding problems were designed to address the emf in analogous ways in the three different contexts. In each case, the existing instruction comprised transformed lectures and tutorials, and the newly developed TLSs replaced older tutorials on mentioned topics.

The principal aim of the first problem (What is the work done when moving charges?) was to understand the emf concept in both electrostatics and dc circuits. The first problem comprised two activities, in which we asked students to consider (A1.1) a test charge in an electric field generated by a point charge $Q$; and (A1.2) a charged particle moving in a closed loop. Activity A1.2 is shown in Fig. 1.

The main objective of A1.3 (Fig. 2) was to apply the concepts of force, work, and energy in contexts of electrostatics. In part A of A1.3, the teacher asked and encouraged the students' groups to write in their notebooks how the test charge $q$ moves from one point to another taking into account three aspects: (1) the type of force acting on the test charge $q$; (2) the work done by the force or forces in moving the charge $q$ along the loop from $F$ to
$F$; (3) the effect of the work done on the energy of the system.

The responses of the groups are then shared and analyzed at whole class level. The teacher resolved doubts and summarized the conclusions of section A of A.1.3. According to the data obtained from the students' notebooks and the teacher's diary, the students groups offered two types of arguments:

(i) A few groups put forward arguments based on electrostatics that justify that the work done by a Coulombic (conservative) force along a closed path is zero. Therefore, if only conservative forces act on the charge $q$, its energy does not vary when making a closed path.

(ii) The explanations of most groups show lack of understanding of the types of force that could act in the context of the activity. Students are unable to distinguish between Coulombic (conservative) electric forces and other possible forces.

In all classroom discussions, the teacher commented on examples studied in electrostatics that support the arguments of type (i). She analyzed that if there were nonconservative forces external to the system, they would change the energy of the system over time along the closed path. Therefore, the effect of the work of these nonconservative forces on the total energy change must be zero.

Next, the students analyzed part B of the activity. According to the data from the students' notebooks and the instructor's diary, almost all groups correctly explained that if there is some energy loss between $G$ and $H$ and some energy gain between $E$ and $F$, it is not possible to explain the energy balance without introducing external nonconservative forces acting on the charge. The vast majority of

\begin{abstract}
I. Energy in a de circuit A de circuit consists of a Van de Graaff generator that pulls electrons from one electrode, making it positive, and pushes them onto the other electrode, a bulb and connecting wires.
\end{abstract}

A. What force(s) act on the electron inside the Van de Graaff generator? Which are conservative, and which are nonconservative?

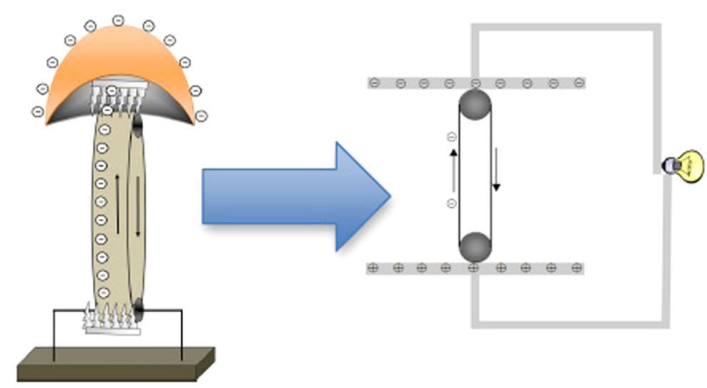

B. What is the work done in moving an electron from one electrode to the other?

C. Use your results from Part III to find the energy balance for the entire circuit.

FIG. 2. Activity A2.1 designed to help students to understand the cause of the movement of charges into the battery and the concept of emf in dc circuits. 
II. Potential difference and emf

A student connects a number of resistors to a battery, one at a time. Each time, she measures the current $i_{R}$ through and the potential difference $V_{R}$ across the resistor. The data are tabulated and graphed.

$\begin{array}{ccc}R(\Omega) & i_{R}(\mathrm{~A}) & V_{R}(\mathrm{~V}) \\ 33 & 0.209 & 6.91 \\ 47 & 0.158 & 7.42 \\ 68 & 0.115 & 7.85 \\ 100 & 0.082 & 8.18 \\ 150 & 0.056 & 8.44 \\ 220 & 0.039 & 8.61\end{array}$

A. Discuss how $i_{R}$ and $V_{R}$ are related to the current $i_{b}$

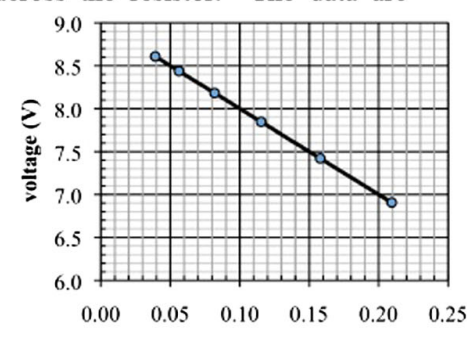
through the battery and the potential difference $V_{b}$ across the battery.

B. Use the graph to determine the emf and the internal resistance of the battery. Clearly explain your reasoning.

FIG. 3. Activity designed to help students to understand that emf and potential difference are different concepts. Students are guided to pay attention to the constant value of the emf of the battery and the dependence of the value of the potential difference on the circuit topology and the current.

the groups used the arguments that have been discussed in the conclusions of part A.

The aim of the second guiding problem (How can you explain and measure the work done in moving charges in a dc circuit?) was to use the emf and potential difference concepts in dc circuits. The second problem of TLS1 comprised three activities, all related to dc circuits: (A2.1) movement of charges in a dc circuit, (A2.2) contrasting potential difference and emf of a battery, and (A2.3) determining the emf of a battery. The activities were designed to help students define the emf concept and distinguish it from potential difference. Figure 3 shows activity A2.1 and Fig. 4 shows A2.2.
According to the data collected from the students' notebooks and the teacher's diary, in A2.1 the vast majority of student groups adequately explained that two types of forces act on the electrons in the Van de Graaff generator, the Coulombic forces of attraction and repulsion between charges, and nonconservative forces exerted by the conveyor belt that are responsible for bringing the electrons to the metallic sphere. The arguments are based on those explained in activity A1.2. In the whole-class discussion, student questions focused on how the Van de Graaff works and how charges are accumulated on the metal sphere. In particular, the teacher encouraged them to reflect on limiting cases of the analogy, such as considering that

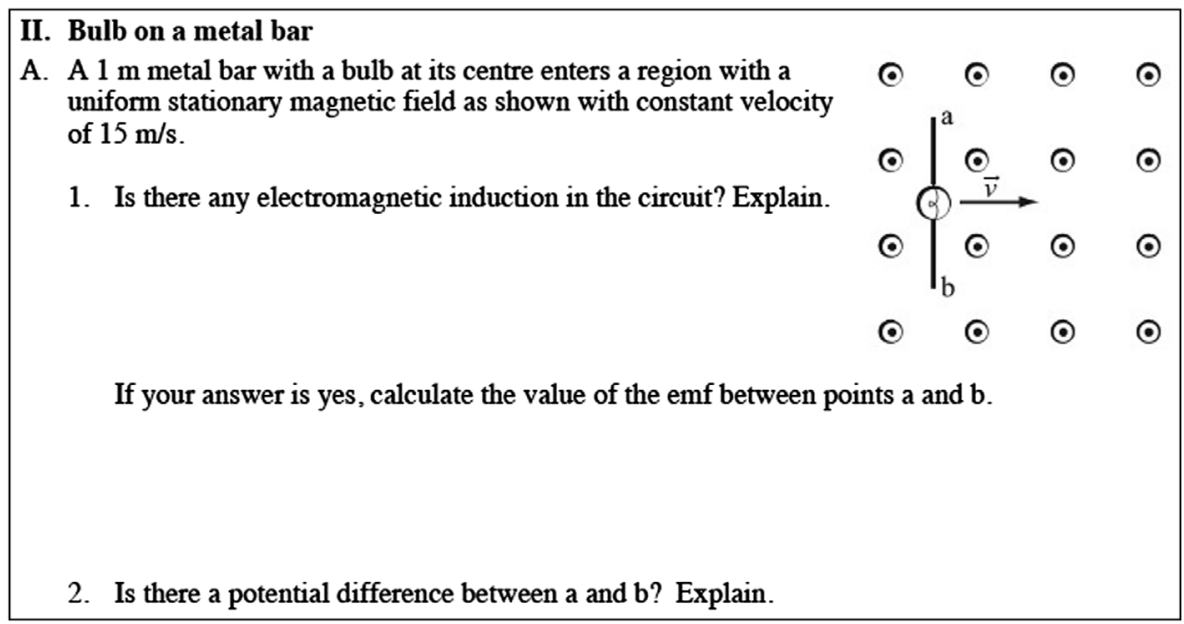

FIG. 4. Activity A.3.2 where the cause of emf is motional. In this case, because the circuit is open the emf generated sets up a potential difference due to charge separation between points $a$ and $b$. 
there is no friction on the belt of the Van de Graaff generator and that the battery has no internal resistance.

In activity A2.2 students were asked to distinguish between emf and potential difference in the context of an experiment. In this activity, students are invited to confront their arguments with experimental data. According to the data obtained from the students' notebooks, some groups use Kirchhoff's loop law $\left[\Delta V_{b}+\Delta V_{R}=0 \times\right.$ $\left(\Rightarrow \varepsilon\right.$-ir $\left.\left.=\Delta \mathrm{V}_{R}\right)\right]$ to establish the relationships between the data from the experiments and the current flowing through the circuit. They apply the law to two measurements in the table and obtain the values of emf and internal resistance of the battery. These groups of students argue based on the data from the experiments and their relation to the theory (Kirchhoff's loop law). In sharing the explanations of the groups with the class, the instructor insisted on the difference between the emf of the battery and the potential difference between its terminals. To end the activity, the instructor proposed students to analyze a situation where the internal resistance of the battery is negligible $(r=0)$ and to indicate if there are differences in the value of the emf and the potential difference of the battery.

The principal objective of the third problem of TLS1 (What happens in an inductive current circuit?) was set in an EMI context. It was given after interactive lectures on electromagnetic induction. It again consisted of three activities: (A3.1) Circuit with a bulb, (A3.2) bulb on a metal bar, and (A3.3) ring around a solenoid. A3.1

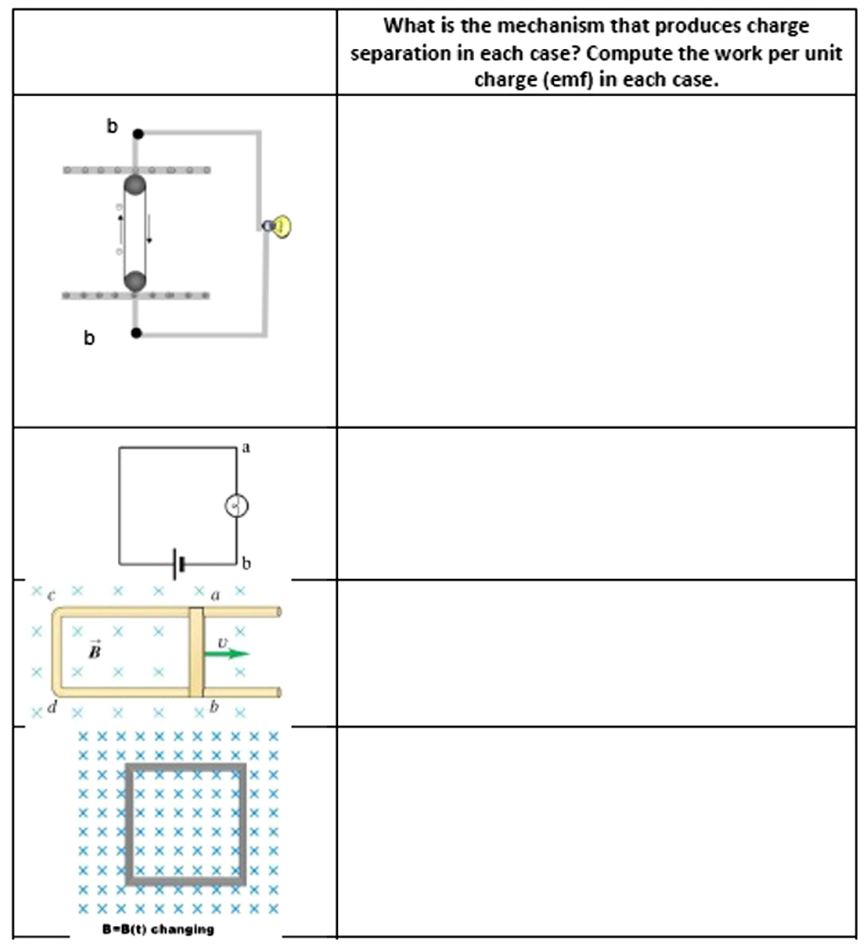

FIG. 5. Worksheet of activity A3 of TLS2 in which students complete the worksheet. contained activities that helped students relate the dc and EMI contexts and helped them distinguish between emf and potential difference. In A3.2 and A3.3, the causes of emf were the movement or motion of a conductor in a magnetic field (A3.2) and a variable magnetic field (A3.3). We present activity A3.2 (see Fig. 5) where the students found problems to distinguish between the concepts of emf and potential difference. The vast majority of student groups argued that it was an induction phenomenon and that there was therefore no potential difference. The teacher had to remind them that charges of different signs accumulated at the ends of the bar due to the Lorentz force. This separation of charges implied that there would be a potential difference between the ends of the bar. The teacher presented the students with situations from the electrostatics chapter that had been worked on in class.

We will present the students' attainment of the learning objectives after the implementation of TLS1 in detail in the next section. However, from data of teachers' diaries we note some key results here:

1. Even after designing instruction specifically to tackle the issue in the third part of the TLS1, the question related to $\mathrm{K} 4$ (emf is not context dependent) was poorly answered.

2. Students in the experimental group outperformed those in the control group.

3. Based on the teachers' diaries and students' notebook, they stated that there were some teaching problems with TLS1:

3.1. The contexts of some of the activities are similar to those encountered in the classroom during the study of the program, for example the analogy of the "mechanical battery" or the movements of bars in a magnetic field. This sometimes produced a loss of student motivation as time went on, and it took too much time.

3.2. Following the previous comment, the decision to address emf analogously in three different settings did not work out in practice as intended. The teachers proposed to present multiple contexts (emf sources or circuits) in all activities, without separating the activities in topics of electrostatics, $\mathrm{dc}$ circuits, and electromagnetic induction.

3.3. Discussions with students and feedback focused mainly on the meaning and role of four elements: charge separation, potential difference, emf and current, in two contexts: dc circuits and EMI.

Taking into account these results a redesign process started. All learning objectives were retained, but the activities of TLS were changed. The changes have focused specifically on the elements mentioned in point 3.3.

Second version of the TLS (TLS2).-Based on the feedback from teachers and students, we decided to shorten the TLS to a single $1.5 \mathrm{~h}$ slot, focusing on helping students to understand that emf is a unifying concept. The second TLS comprised four activities centered on one main 


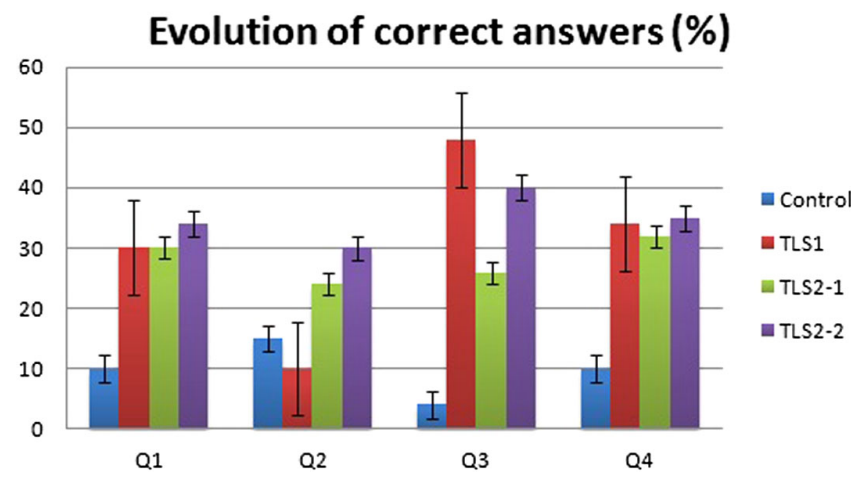

FIG. 6. The evolution of the prevalence of answers in category A (correct and underpinned by reasoning in accordance with the epistemology of science) for all the questions for control and experimental groups.

problem (How to measure the work done when moving charges in different contexts?). In each activity, four different emf sources or circuits were presented all together in a single guided inquiry worksheet to be filled by the students in groups. The questions were designed to help students to attain the learning objectives. In activity A1, we presented different kinds of emf sources and asked the students for each case if charge separation, potential difference, emf and/or current occur. In activity A2, we asked the students about the mechanism that produces charge separation in different emf sources. In activity A3 (see Fig. 6), we connected the sources of emf to a circuit with a resistor and asked the students the same questions as in activity A1. Finally, in activity A4 the students were asked whether there are differences if the source of emf is ideal or real, and whether that affects the numerical values of the emf and the potential difference.

Activity 3.-The data from the student's notebooks and the teacher's diary shows that the students understand the questions and the teacher intervenes very little during the work of the student groups. In the first two cases, almost all groups argue that the battery produces a separation of charges and that therefore there is a potential difference between its poles, which causes a current throughout the circuit. Most groups indicate that the work per unit charge carried out by the nonconservative mechanic forces $F_{\mathrm{NC}}$ is calculated as $\varepsilon=W_{F_{\mathrm{NC}}} / q=F_{\mathrm{NC}} L / q$ in the first case and that in the second case, the emf is calculated as $W_{\text {chemical }} / q$. Some groups argue qualitatively but do not write the equations. The teacher encouraged students to quantitatively define the concept of emf in each context.

In the last two cases where the current was produced by EMI, the students' arguments have different explanatory levels:

(1) In the third case, almost all the groups argued based on Faraday's law that the movement of the bar in the magnetic field produces emf and that there is current. A few groups argued that the Lorentz force acts on the electrons of the bar and that therefore charges of different signs accumulate at the ends of the bar, causing a potential difference. A significant percentage of the groups indicated the quantitative equation $\varepsilon=\mathrm{IR}$. The teacher had to insist in her explanations to the whole class that there is a charge separation in the bar and analyze the equation $\varepsilon=\Delta V=I R$. The teacher suggests that students review the examples discussed in the EMI chapter.

(2) In the fourth case, most groups of students based their arguments on Faraday's law (variation of magnetic flux produces emf) to justify their affirmative answer to the existence of emf and induced current, indicating $\varepsilon=\mathrm{IR}$. However, none of these groups of students argued that there is no potential difference or separation of charges. The teacher had to insist that they apply the Lorentz force on the electrons of the wire and check that they move (there is current) but that there is no accumulation of charges and therefore there is no potential difference.

This second version was implemented twice in the University of the Basque Country (UPV/EHU) during 2015-2016 $(n=50)$ and 2016-2017 $(n=49)$ under the same conditions. The same experienced teacher taught TLS2 at the end of the course, i.e., on completion of the lectures on electromagnetic induction (even though dc circuits were also included). We note that UPV/EHU introductory engineering students are used to follow active leaning lectures in physics as explained at the beginning of this section. We were prepared to see less or no improvement on questions probing the other key characteristics, but the students' responses were surprisingly similar to that of the cohort who experienced TLS1. Moreover, the question related to the context dependency was answered a little better.

\section{RESULTS AND ANALYSIS OF THE TLSS}

To analyze the effectiveness and the quality of the tutorials we used the same phenomenographical approach $[34,36]$ as in our previous work to establish the postinstruction attainment of the learning objectives [14,15]. We selected three questions from these papers which were already validated (see Table III), plus the question shown in Fig. 1 which was similarly validated by colleagues and a small number of students.

As mentioned before, we take the cohorts of UPV/EHU students in our previous work as the control group, and the groups that followed TLS1 (2014-2015) and TLS2 (2015-2016 and 2016-2017) as three experimental groups. Thus, we compare the results after implementing the TLSs with the previous results after regular instruction (i.e., in a reformed setting but with end of chapter problems instead of a TLS). Although we did not ask pretest questions specific to emf, we consider the cohorts equivalent as they all have experienced the same introductory physics 
TABLE III. Open ended questions used to analyze students' attainment of the learning objectives. See Appendix.

\begin{tabular}{|c|c|c|}
\hline & Question & Learning objective \\
\hline Q1 & dc circuit (Q3 from Garzón et al. [14] & $\begin{array}{l}\text { Ask students to analyze energy balance of an electrical circuit } \\
\text { made up of a battery, wires, and a resistor (LO5). They have to } \\
\text { recognize the emf as the work done per unit of charge by } \\
\text { nonconservative forces (LO1) and the potential difference as } \\
\text { that done by conservative (LO3) }\end{array}$ \\
\hline Q2 & Two circuits EMI and dc & $\begin{array}{l}\text { Ask students to recognize that electromotive force is } \\
\text { a cross-cutting concept across different contexts (LO2) }\end{array}$ \\
\hline Q3 & Magnet and coil (Q1 from Zuza et al. $[15])$ & $\begin{array}{l}\text { Probing students' ability to distinguish between emf and } \\
\text { potential difference (LO4) in a familiar electromagnetic } \\
\text { induction context. }\end{array}$ \\
\hline Q4 & Half ring (Q3 from Zuza et al. [15]) & $\begin{array}{l}\text { Probing students' ability to recognizing work done } \\
\text { by Lorentz force (nonconservative) (LO1, LO6) and } \\
\text { distinguish between emf and potential difference (LO4). }\end{array}$ \\
\hline
\end{tabular}

curriculum, and their examination marks did not significantly differ.

Regarding the conceptual and epistemic difficulty of the questions, all of them are familiar to students in the academic context (see Appendix). However, the questions Q1 and Q4 are not similar to those at the end of the chapter problems. In these two questions students have to use a more complex reasoning that have to apply the concepts of emf and potential difference in transitory situations (open circuit in Q1 and variable magnetic field in Q4).
The first step of the phenomenographical research [36] showed that the categorization obtained for students' difficulties $[14,15]$ is also suited to categorize students' post-TLS answers. Figure 6 shows the evolution of the prevalence of answers in category A from control group (C) to the group that attended the first version of the TLS, TLS1, and both groups that attended the second version of the TLS, TLS2. Figure 7 shows the same data for all categories.

We can see that answers in category A are more prevalent in all experimental groups, except for question 2 for TLS1.

\section{Question 1}

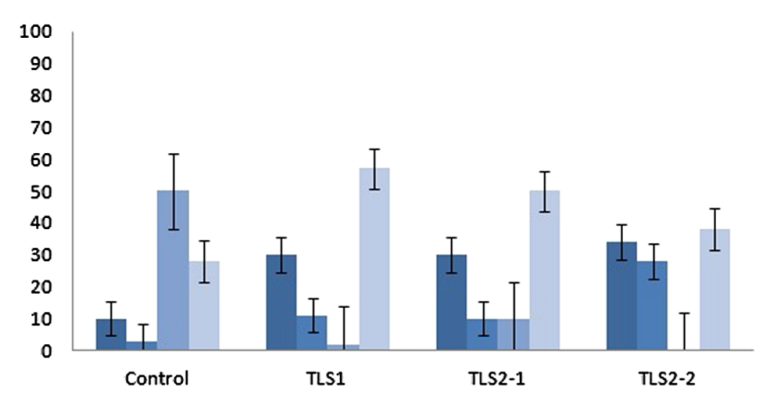

Question 3

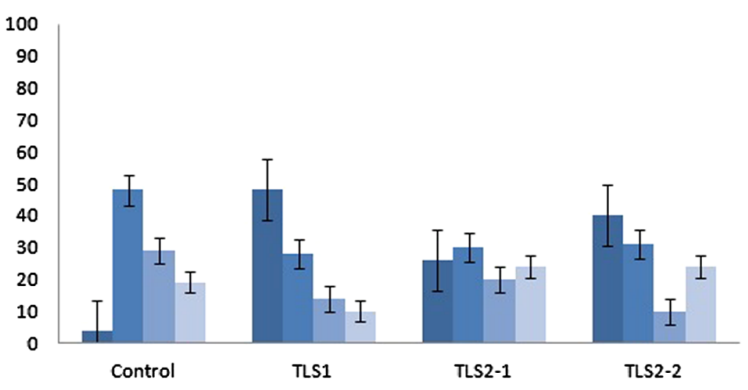

Question 2

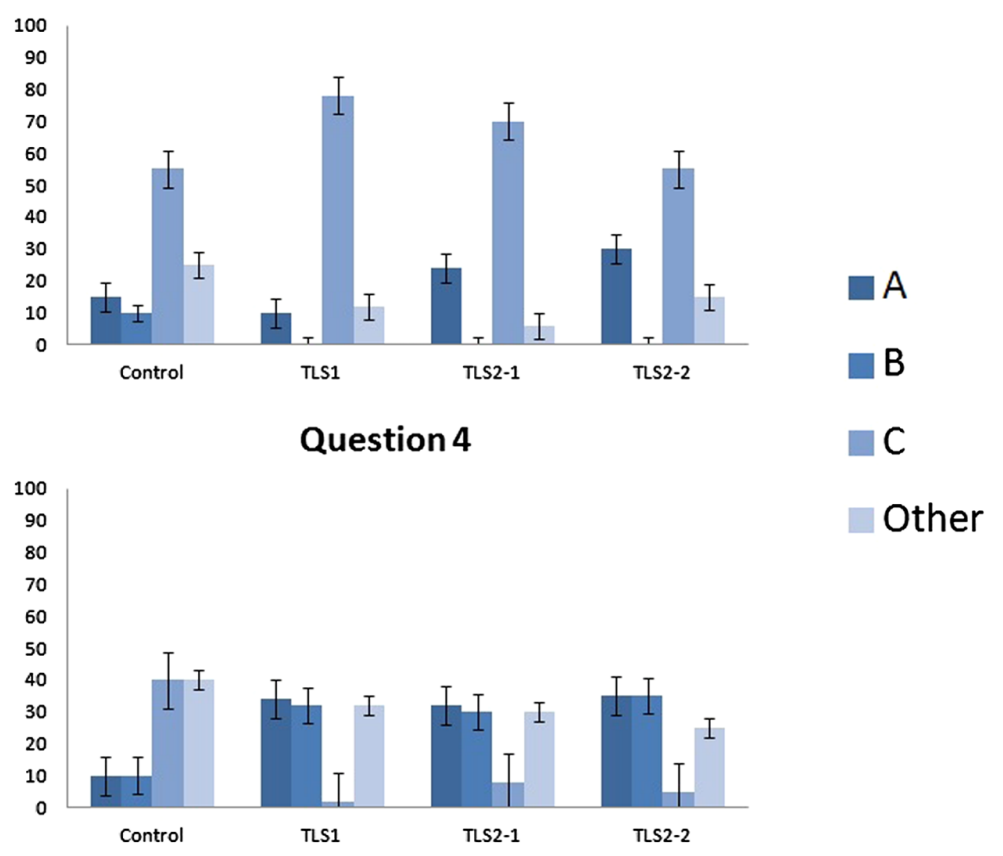

FIG. 7. Prevalence of responses (in percent) to four questions by students in the control group, the group that followed TLS1, and the groups that followed TLS2 (TLS2-1 and TLS2-2). Category A comprises correct answers based on scientific explanations. Answers in category B use scientific arguments but answer the question incorrectly or incompletely. In category C nonscientific explanations or descriptions of the phenomena are included. The "others" category comprises incoherent and blank answers. 


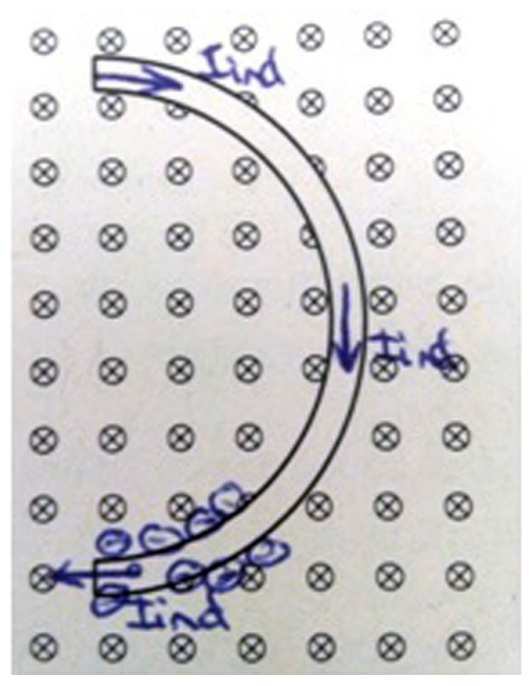

FIG. 8. Drawing of a student (TLS2-1) who correctly answered the half-ring question.

We found no qualitatively different quotes for answers in category A to those already published [14,15]. The following quotes illustrate typical answers in this category:

"When the circuit is closed, the efficiency of the battery is no longer $100 \%$ due to internal resistance, there are losses that are going to make the potential difference lower than emf" (Q1, TLS1)

"There is emf in both circuits and they are the same thing. The difference between them is how they are generated. In the figure on the left there is a battery and in the second figure there is a changing field that generates current so, there is no battery needed for an emf. Despite this they are equal and the bulb will light in both cases" (Q2, TLS2-2)

"There won't appear any charge separation in the ring so there is no Coulombic electric field nor a potential difference. In this case the current will be generated by a nonCoulombic electric field" (Q3, TLS2-1)

"As the circuit is not closed, the changing magnetic field will generate an induced transient current that I draw [see Fig. 8] due to a changing magnetic flux $I=\varepsilon / R$. The electrons will go down so a potential difference will appear" (Q4, TLS2-1)

Answers in this category B are typically incomplete rather than totally incorrect. There is a focus on the force concept and students give it the capability to do "work" to move charges, but they do not link emf and potential difference. The following quotes illustrate typical answers in this category:

"The correct equation is option (b) since it is the electromotive force that pushes the electrons throughout the circuit including the passage through the battery" (Q1, TLS1)

"There is emf in both circuits and they are the same thing. The emf is the force that acts on the electrons, difference between two context is that in the first one it is a direct current circuit and in the second one it is an electromagnetic induction circuit" (Q2, TLS2-1)

'I do not agree. The current is not generated by the potential difference. It is generated by the magnetic field.' (Q3, TLS2-2)

"The changing magnetic field will generate an induced transient current due to a changing magnetic flux $I=$ $\varepsilon / R$. So there is emf, but not potential difference (Q4, TLS2-1)

We used the two tailed exact Fisher test for statistical data analysis [37]. For questions Q1, Q3, and Q4 there are statistical differences $(p<0.05)$ for all the TLS implementations in comparison with the control group, while there are no statistical differences between the three experimental groups regarding the prevalence of answers in category A. For Q2 there is no statistical difference between the control group and the first version of the TLS $(p=0.4)$, but there is a significant difference between T1 and T2 $(p<0.05)$.

Regarding the evolution of the other categories, we see that for the more complex questions $(\mathrm{Q} 1$ and $\mathrm{Q} 4)$ more students use isolated elements from a scientific framework (category B), so there are now more students with partial understanding [37]. For question Q3 fewer students in the experimental groups use isolated elements from a scientific framework or mix up emf and potential difference (category C) than in the control group, while correct answers (category A) are more prevalent. We note that some students in the control group already showed scientific conceptions corresponding to emf in EMI contexts, but did not distinguish between emf and potential difference. It seems that the TLSs help students to go a step further and get a better comprehension of emf and how it is different from potential difference.

\section{DISCUSSION AND CONCLUSIONS}

We have described the design and implementation of two TLSs developed within a transformed course setting that targeted students' learning understanding concerning emf. Guided by the identification of six key characteristics of emf we wanted our students to understand and a commitment to active learning strategies, we developed two TLSs. In TLS1, we devoted three 1.5-h tutorial slots to helping students attain the learning outcomes at apparently appropriate times. On completion of electrostatics, the students had encountered all the material needed to distinguish between work done by conservative and nonconservative forces; on completion of dc circuits, they were in a position to apply this distinction in de circuits and thereby distinguish between emf and potential difference; and on completion of electromagnetic induction, they were ready for a tutorial that brought all elements together.

During implementation it became clear that TLS1 was beneficial to students in terms of improved attainment of the learning outcomes as they outperformed the control group, but at the same time teachers reported that the 
intervention took a lot of time and that students found the activities repetitive. We decided to eliminate the first two tutorials and created a TLS2 that only comprised a revised tutorial implemented on completion of all material. As mentioned before, in TLS2 the role of emf as a crosscutting concept was a central idea. Student attainment on completion of TLS2 was similar to that on completion of TLS1.

One important aspect of DBR based research is the redesign of the TLS. This aspect is not usually reported outside the specialist DBR literature, but it is an important characteristic of our research. The analysis of the results obtained from the implementation of TLS1 has given rise to a new sequence that is substantially different from the first, but resulted in similar student attainment. Thus, we advocate implementation of TLS2, which is much shorter and thereby easier to implement.

It should be remembered that the implementation of the TLSs has been carried out in introductory physics groups that are used to working in groups within a transformed physics course. We do not have data on how this TLS could work in students receiving traditional education. However, based on our teaching experience, we think that our design can be useful for those students who have experience in learning with active teaching methodologies such as "Peer Instruction" or "tutorials." These teaching strategies train students to formulate hypotheses, argue their proposals, and discuss results based on data. Therefore, these students will find familiar the way of asking the problems and trying to solve them, as the two designed TLS propose. One of the central ideas in the scientific paradigm is replicability; however, because research based on TLS designs cannot (and may not want to) manipulate school contexts, it is difficult to replicate the findings in contexts where instructional methodology is very different. For this reason, we think that our design of TLSs will not work in teaching contexts where students mainly listen to the teacher and take notes. We think that these students are not familiar to learning the concepts and laws along with practicing scientific skills, which requires the resolution of the TLS activities.

\section{ACKNOWLEDGMENTS}

We are grateful to Ricardo Karam and Lana Ivanjek for thorough discussions of this paper. Part of this research was founded by the Spanish government (MINECOI FEDER Project No. PID2019-105172RB-I00) and by the University of the Basque Country (GIU19/071).

\section{APPENDIX}

The following questions make up the questionnaire that has been used to measure the learning that students achieved on the key ideas defined in the TLSs. The questionaire has four questions (Figures 9-12).

Question 1.

A battery with an emf of $12 \mathrm{~V}$ and an internal resistance of $200 \Omega$ is placed in series with a $1000 \Omega$ resistor as shown in the figure.

A) When the switch is open, the potential difference between points $a$ and $b$ of the battery will be:

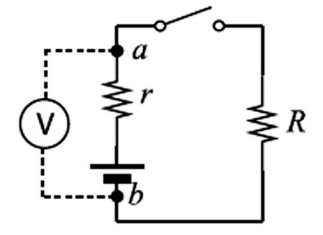

a) Equal to $12 \mathrm{~V}$

b) Greater than $12 \mathrm{~V}$

c) Less than $12 \mathrm{~V}$

Explain your answer and indicate how you would calculate the potential difference.

B) When the switch is closed and there is a stationary current $I$, the potential difference between terminals $a$ and $b$ of the battery will be:

a) Equal to $12 \mathrm{~V}$

b) Greater than $12 \mathrm{~V}$

c) Less than $12 \mathrm{~V}$

Explain your answer and indicate how you would calculate the potential difference.

FIG. 9. Question 1. 
Question 2.

Figure 1 shows a continuous current electric circuit with a 12 volt battery as the electromotive force. Figure 2 shows an induced current circuit that has an electromotive force of 16 volts.

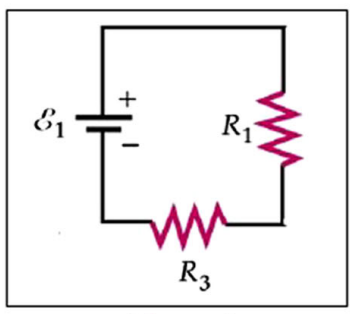

Figure 1

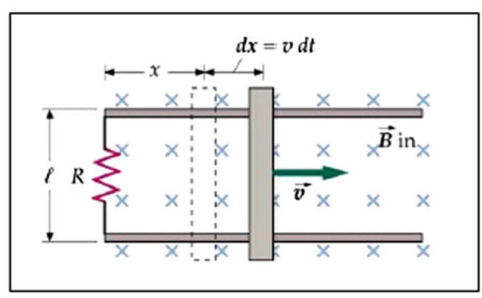

Figure 2

A student states that "Although in both cases the emf is expressed in the same unit, the two electromotive forces respond to two different physical concepts".

Do you agree with student's answer? Argue carefully by explaining the physical meaning of the emf in both situations.

FIG. 10. Question 2.

Question 3.

When a magnet travels at constant speed closer towards a loop at rest (see diagram), an electric current is induced in the loop. Explain whether the following statement is correct:

"A potential difference has been induced and therefore an electric current appears in the ring."

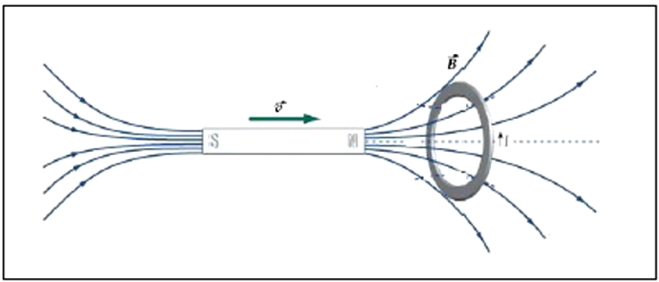

FIG. 11. Question 3.

Question 4.

Consider a conducting half-ring placed in a uniformly increasing magnetic field.

When the situation is stationary in the half-ring, is there a potential difference? If so, explain how.

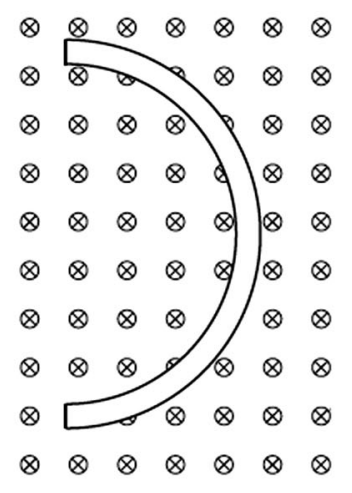

FIG. 12. Question 4. 
[1] P. A. Tipler and G. Mosca, Physics for Scientists and Engineers (Macmillan London, 2007).

[2] P. M. Fishbane, S. G. Gasiorowicz, and S. T. Thornton, Physics: For Scientists and Engineers with Modern Physics (Prentice-Hall Englewood Cliffs, NJ, 2005).

[3] H. D. Young, R. A. Freedman, and A. L. Ford, University Physics with Modern Physics (Pearson, Boston, MA, 2015), p. 1600.

[4] L. C. McDermott and P. S. Shaffer, Research as a guide for curriculum development: An example from introductory electricity. Part I: Investigation of student understanding, Am. J. Phys. 60, 994 (1992).

[5] D. P. Smith, and P. van Kampen, Teaching electric circuits with multiple batteries: A qualitative approach, Phys. Rev. ST Phys. Educ. Res. 7, 020115 (2011).

[6] P. Mulhall, B. McKittrick, and R. Gunstone, A perspective on the resolution of confusions in the teaching of electricity, Res. Sci. Educ. 31, 575 (2001).

[7] J. Guisasola, J. M. Almudi, and K. Zuza, University students' understanding of electromagnetic induction, Int. J. Sci. Educ. 35, 2692 (2013).

[8] M. R. Stetzer, P. van Kampen, P. S. Shaffer, and L. C. McDermott, New insights into student understanding of complete circuits and the conservation of current, Am. J. Phys. 81, 134 (2013).

[9] R. Chabay and B. Sherwood, Restructuring the introductory electricity and magnetism course, Am. J. Phys. 74, 329 (2006).

[10] K. Zuza, J. M. Almudí, A. Leniz, and J. Guisasola, Addressing students' difficulties with Faraday's law: A guided problem solving approach, Phys. Rev. ST Phys. Educ. Res. 10, 010122 (2014).

[11] H. V. Mauk and D. Hingley, Student understanding of induced current: Using tutorials in introductory physics to teach electricity and magnetism, Am. J. Phys. 73, 1164 (2005).

[12] S. Stocklmayer and D. Treagust, A historical analysis of electric currents in textbooks: A century of influence on physics education, Sci. Educ. 3, 131 (1994).

[13] F. Reif, Generalized Ohm's law, potential difference, and voltage measurements, Am. J. Phys. 50, 1048 (1982).

[14] I. Garzón, M. De Cock, K. Zuza, P. Van Kampen, and J. Guisasola, Probing university students' understanding of electromotive force in electricity, Am. J. Phys. 82, 72 (2014).

[15] K. Zuza, M. De Cock, P. van Kampen, L. Bollen, and J. Guisasola, University students' understanding of the electromotive force concept in the context of electromagnetic induction. Eur. J. Phys. 37, 065709 (2016).

[16] L. C. McDermott and P.S. Shaffer, Tutorials in Introductory Physics (Prentice Hall, Englewood Cliffs, NJ, 1998).

[17] D. Gil and and J. Carrascosa, Bringing pupils' learning closer to a scientific construction of knowledge: A permanent feature in innovations in science teaching, Sci. Educ. 78, 301 (1994).

[18] M. Meheut and D. Psillos, Teaching-learning sequences: Aims and tools for science education research, Int. J. Sci. Educ. 26, 515 (2004).
[19] J. Guisasola, K. Zuza, J. Ametller, and J. GutierrezBerraondo, Evaluating and redesigning teaching learning sequences at the introductory physics level, Phys. Rev. Phys. Educ. Res. 13, 020139 (2017).

[20] M. W. Easterday, D. R. Lewis, and E. M. Gerber, Designbased research process: Problems, phases, and applications (International Society of the Learning Sciences, Boulder, CO, 2014), pp. 317-324.

[21] J. Kalle, J. Lavonen, and V. Meisalo, Pragmatic Designbased research-Designing as a shared activity of teachers and researches, Iterative Design of Teaching- Learning Sequences (Springer Netherlands, Dordrecht, 2016), pp. 35-46.

[22] J. Martinez-Torregrosa, R. Limiñana, A. Menargues, and R. Colomer, In-depth teaching as oriented-research about seasons and the sun/hearth model: Effects on content knowledge attained by pre-service primary teacher, J. Baltic Sci. Educ. 17, 97 (2018), http://oaji.net/articles/ 2017/987-1519203761.pdf.

[23] C. Furió, J. Guisasola, J. M. Almudi, and M. Ceberio, Learning the electric field concept as oriented research activity, Sci. Educ. 87, 640 (2003).

[24] J. A. Acevedo-Díaz, Á. Vázquez-Alonso, M. A. ManasseroMas, and P. Acevedo-Romero, Consensos sobre la naturaleza de la ciencia: fundamentos de una investigación empírica, Revista Eureka sobre Enseñanza y Divulgación de las ciencias 4, 42 (2007).

[25] M. R. Matthews, Science Teaching: The Role of History and Philosophy of Science (Routledge, London, 1994).

[26] N. J. Nersessian, Should physicists preach what they practice?, Sci. Educ. 4, 203 (1995).

[27] H. Härtel, The electric circuit as a system: A new approach, Eur. J. Sci. Educ. 4, 45 (1982).

[28] R. N. Varney and L. H. Fisher, Electromotive force: Volta's forgotten concept, Am. J. Phys. 48, 405 (1980).

[29] R. W. Chabay and B. A. Sherwood, Matter and Interactions (John Wiley \& Sons, New York, 2011).

[30] R. H. Romer, What do "voltmeters" measure?: Faraday's law in a multiply connected region, Am. J. Phys. 50, 1089 (1982).

[31] J. Guisasola, Teaching and learning electricity: The relations between macroscopic level observations and microscopic level theories, in International Handbook of Research in History, Philosophy and Science Teaching (Springer, Dordrecht 2014), pp. 129-156.

[32] R. W. Chabay and B. A. Sherwood, Matter and Interactions (John Wiley \& Sons, New York, 2015).

[33] P. Cobb, J. Confrey, A. DiSessa, R. Lehrer, and L. Schauble, Design experiments in educational research, Educ. Res. 32, 9 (2003).

[34] F. Marton, Phenomenography-describing conceptions of the world around us, Instr. Sci. 10, 177 (1981).

[35] P. M. Fishbane, S. Gasiorowicz, and S. T. Thornton, Physics for Scientists and Engineers, 2nd ed. (PrenticeHall, Englewood Cliffs, NJ, 1996), p. 839.

[36] F. Marton and S. Booth, Learning and Awareness (Lawrence Erlbaum, Hillsdale, NJ, 1997).

[37] P. R. Heron, Effect of lecture instruction on student performance on qualitative questions, Phys. Rev. ST Phys. Educ. Res. 11, 010102 (2015). 